\title{
Evaluation of Donor Site Pain After Fractional Autologous Full-Thickness Skin Grafting
}

\author{
Jose A. Jaller, Ingrid Herskovitz, Luis J. Borda, Joshua Mervis, \\ Evan Darwin, Penelope A. Hirt, Hadar Lev-Tov, and Robert S. Kirsner* \\ Department of Dermatology and Cutaneous Surgery, University of Miami Miller School of Medicine, Miami, Florida.
}

Background: Despite the development of numerous wound treatment alternatives, $25 \%$ to $50 \%$ of leg ulcers and $>30 \%$ of foot ulcers are not fully healed after 6 months of treatment. Autologous skin grafting is a time-tested therapy for these wounds; however, the creation of a new wound in the donor area yields a considerable limitation to this procedure.

Innovation: Fractional autologous full-thickness skin grafting (FFTSG) is a technique wherein multiple small full-thickness skin grafts (FTSGs) are harvested with possibly minor donor-site comorbidities. The first device used to harvest FFTSG (ART ${ }^{\mathrm{TM}}$ system, Medline, Northfield, IL) is a device capable of harvesting $>300$ small FTSGs and transferring them to a target wound.

Objective: To better evaluate patients' clinical experience, we sought to evaluate pain at the donor site associated with this procedure.

Approach: Pain was assessed with numeric visual analog pain scales at days $1,2,4$, and 7 . Nine subjects underwent this procedure with only six of them reporting any level of pain on day 1 , and none disclosing pain after day 2 .

Conclusion: In this study, we evidenced that this device manages to harvest FTSGs with minimal associated pain. Future research will need to evaluate other aspects of the procedure as well as long-term outcomes at the donor and recipient areas.

Keywords: skin graft, fractional autologous full-thickness skin graft, chronic ulcers, acute wounds, pain

\section{INTRODUCTION}

WOUNDS, DEFINED AS a disruption of the skin that results in a loss of function or skin architecture, typically heal in a predictable and timely manner. ${ }^{1}$ However, under certain conditions, wounds may persist and become chronic. Venous leg ulcers (VLUs), diabetic foot ulcers (DFUs), and pressure ulcers are the most common etiologies of chronic wounds. VLUs account for $70 \%$ of leg ulcers, ${ }^{2,3}$ whereas DFUs are the most common foot ulcer and often result in amputation. ${ }^{4}$
Skin injury from ulcers and burns comprises a significant public health concern, with a reported prevalence of $4.64 \%$ in the U.S. population and an estimated cost of $>\$ 6$ billion annually. ${ }^{5}$ In addition, wounds and burns are responsible for $>15 \%$ of all skin disease-related deaths, accounting for $\sim 3,453$ deaths in $2013 .{ }^{5}$ With the projected increase in the life span of the U.S. population, new therapeutic approaches will be needed to respond to this growing need.

(c) Jose A. Jaller et al. 2018; Published by Mary Ann Liebert, Inc. This Open Access article is distributed under the terms of the Creative Commons Attribution Noncommercial License (http:// creativecommons.org/licenses/by-nc/4.0), which permits any noncommercial use, distribution, and reproduction in any medium, provided the original authors and the source are cited.

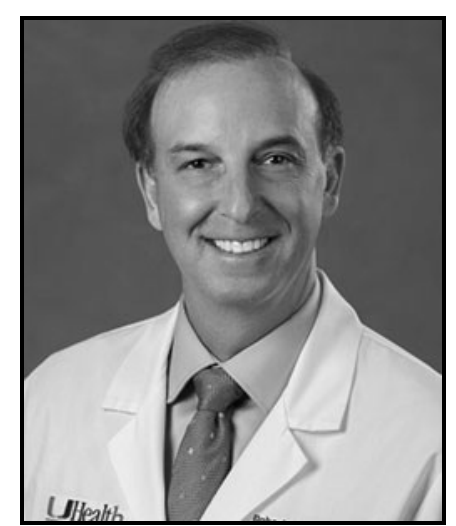

Robert S. Kirsner, MD, PhD

Submitted for publication May 15, 2018. Accepted in revised form May 22, 2018.

${ }^{*}$ Correspondence: Department of Dermatology and Cutaneous Surgery, University of Miami Miller School of Medicine, 1600 NW 10th Avenue, Rosenstiel Medical Science Building-Room 2023A, Miami, FL 33136

(e-mail: Rkirsner@med.miami.edu). 
These hard-to-heal wounds may be amenable to autologous skin grafting, a time-tested therapy considered one of the most efficacious in wound care. ${ }^{6}$ Kirsner et al. hypothesized 25 years ago that autologous skin grafts work not only by tissue replacement but also as active pharmacologic instruments that stimulate wound healing. ${ }^{7}$ Autologous skin grafts are classified by the depth of the skin harvested. Epidermal skin grafts (ESGs) are constituted only by the epidermis of the skin. The harvest is performed with a device that uses thermal-regulated suction chambers to generate an array of small epidermal blisters. This procedure takes place in an outpatient setting and requires no local anesthesia. In addition, the donor site heals as early as 2 days after grafting and causes virtually no pain or scarring in the donor area. ${ }^{8,9}$ In contrast, the absence of dermis in the harvest confers disadvantages such as the lack of fibroblasts in the graft, the little tensile strength provided to the treatment wound, and the diminished functionality of the new skin. ${ }^{10}$ Since ESGs have limited donor site morbidity, this procedure should be considered as an alternative to other autologous skin grafts when restoration of skin function is not imperative and the wound is superficial and small.

Split-thickness skin grafts (STSGs) are composed of the complete epidermis and variable amounts of the dermis. They can be divided into thin, medium, and thick STSGs depending on the quantity of dermis included. Despite lack of randomized controlled trial data, STSGs have been considered standard of care for the reconstruction of large wounds. ${ }^{11}$ The donor tissue can undergo two techniques, meshing and expansion micrografting, ${ }^{10}$ that expand the graft allowing its use in large wounds while sacrificing function and cosmetic outcomes. ${ }^{6}$ Despite not extracting the full dermis, STSGs are associated with significant donor site complications common to other grafting techniques.

Full-thickness skin grafts (FTSGs) comprise epidermis and the entire dermis. Currently, this technique is the only treatment option capable of fully restoring dermal components. ${ }^{12}$ This procedure provides the highest wound tensile strength with the least contracture and superior functional and cosmetic results. ${ }^{8}$ Traditionally, FTSGs help reduce wound contraction and improve cosmesis and are used to repair wounds from skin tumor excisions where cosmesis is important or contraction will cause functional issues or reserved for severe burns.

Newly developed fractional autologous fullthickness skin grafting (FFTSG) is a procedure that consists of harvesting multiple, discontinuous, narrow skin columns (i.e. microcolumns) that represent a fraction of a predetermined skin surface area. Traditional techniques used for FTSGs and STSGs create large wounds that heal with scarring, producing dysfunctional, distressing, and aesthetically compromised skin. By contrast, fractional micrometric wounds heal by remodeling, a process characterized by a full reconstruction of normal tissue architecture, as well as re-establishment of complete functionality. ${ }^{13}$ FFTSG has recently become available with the newly developed device (Autologous Regeneration of Tissue, ART ${ }^{\mathrm{TM}}$; Medline, Northfield, IL) that can harvest hundreds of small FFTSGs, which aims to minimize complications. This device harvests the entire dermis and epidermis in a micrometric, microcolumn configuration and then transfers the tissue into a target wound.

\section{CLINICAL PROBLEM ADDRESSED}

Despite the development of numerous wound treatment alternatives, $25 \%$ to $50 \%$ of leg ulcers and $>30 \%$ of foot ulcers are not fully healed after 6 months of treatment. ${ }^{3}$ These wounds may be suitable for skin grafting. However, the generation of a new wound at the harvesting site comes with multiple comorbidities including pain, scarring, and risk of infection that represent significant drawbacks to such a procedure. Little is known about this procedure; hence, we sought to evaluate donor site pain associated with it.

\section{MATERIALS AND METHODS}

The device that harvests FFTSG (ART; Medline) consists of two components: an electronic, reusable, handheld device and a disposable cartridge. Each cartridge consists of 20 rows of 21 regular wall gauge needles arranged in a $28.7 \mathrm{~mm}$ diameter circular array. Once a cartridge is loaded into the handheld device, the harvester is capable of harvesting $316 \mathrm{FTSGs}$, each $500 \mu \mathrm{m}$ wide and up to $3.25 \mathrm{~mm}$ deep. When triggered, needles are deployed into the donor site one row at a time. After all 20 rows have been harvested, the needles retract en masse with skin microcolumns retained inside. A total of $10 \%$ of the skin surface area within the circular array is harvested. The harvester is then repositioned at close range over the wound site, and grafts are ejected from the cartridge onto the wound. This harvesting process can be repeated with a single cartridge up to three times per patient, after which the cartridge should be disposed of in a standard "sharps" container. The harvester used for this study was provided by Medline, Northfield, Illinois. 

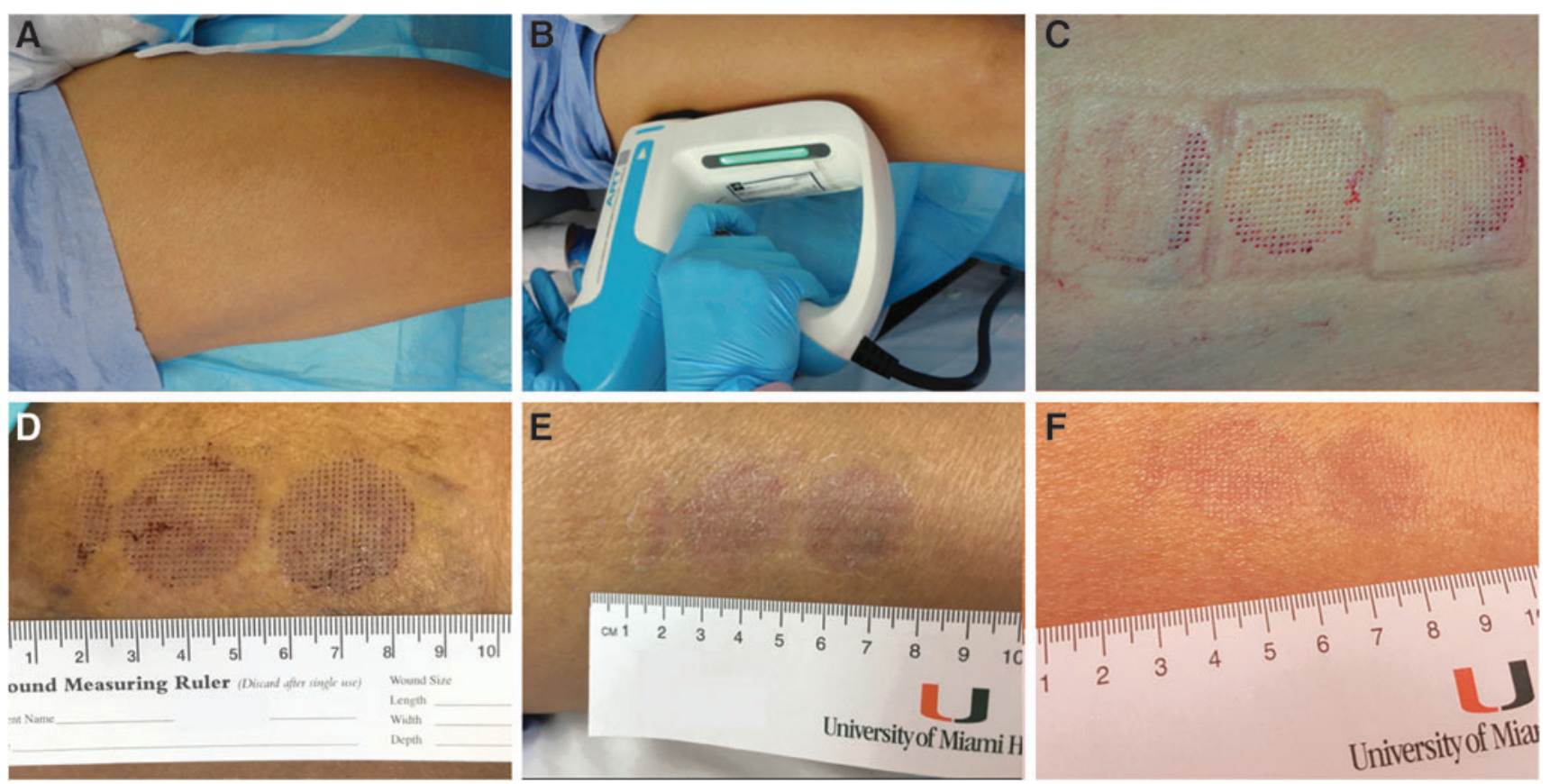

Figure 1. Donor-site harvesting. (A) Lateral thigh before harvesting. (B) Harvesting. (C) Donor-site immediately after harvest. (D) Donor-site 7 days after procedure. (E) Donor-site 23 days after procedure. (F) Donor-site 60 days after procedure.

After informed consent, nine subjects with various wound etiologies were selected to undergo this procedure at our ambulatory care center. In all cases, the donor site was located on the thigh, medial, or lateral to the femur. The donor site was sterilized using alcohol swabs, and a sterile field surrounding the donor site was prepared. Local anesthesia to the donor site was given through injection of lidocaine $\mathrm{HCl} 2 \%$ with epinephrine $1: 100,000$. One to three arrays of microcolumns were harvested from each patient depending on wound size (Fig. 1). A nonadherent pad and film dressing were applied to the donor site postprocedure. Pain at the donor site was then evaluated at 1, 2, 4, and 7 days after grafting. Owing to the anesthesia, pain was not evaluated during or in the immediate hours after the procedure. Pain assessment was performed using a validated visual analog scale (VAS), a continuous scale anchored by

Table 1. Subjects' demographics and wound etiology

\begin{tabular}{lc}
\hline Age & $58(12.9)$ \\
Gender & $3(33.3)$ \\
$\quad$ Men (\%) & $6(66.7)$ \\
$\quad$ Women (\%) & \\
Wound etiology & $6(66.7)$ \\
$\quad$ Venous ulcer (\%) & $1(11.1)$ \\
Surgical/radiation (\%) & $1(11.1)$ \\
Pyoderma gangrenosum (\%) & $1(11.1)$ \\
Sickle cell ulcer (\%) & $1 \%$ \\
\hline
\end{tabular}

"no pain" at 0 and "worst pain imaginable" at $10 \mathrm{~cm}$, overlaid with intermediate numeric marks. ${ }^{14} \mathrm{In}$ addition, new use of pain medications was documented at each follow-up point.

\section{RESULTS}

Nine subjects underwent the FFTSG (Fig. 1). The mean age was 58 years, six $(66.7 \%)$ were women and three (33.3) were men. The most common wound etiology was VLU (66.7\%), followed by surgical/radiation, pyoderma gangrenosum, and sickle cell ulcer with $11.1 \%$ each (Table 1). Of the nine subjects, six reported pain 1 day after the procedure (Table 2). The average VAS score 1 day

Table 2. Subject visual analog scale pain scores in donor-site after treatment

\begin{tabular}{lllll}
\hline & \multicolumn{4}{c}{ Time After Procedure } \\
\cline { 2 - 5 } Subject No. & 1 Day & 2 Days & 4 Days & 7 Days \\
\hline 1 & 0 & 0 & 0 & 0 \\
2 & 2.5 & 1 & 0 & 0 \\
3 & 1 & 0 & 0 & 0 \\
4 & 3 & 2 & 0 & 0 \\
5 & 1 & 0 & 0 & 0 \\
6 & 1 & 1 & 0 & 0 \\
7 & 0.5 & 0 & 0 & 0 \\
8 & 0 & 0 & 0 & 0 \\
9 & 0 & 0 & 0 & 0 \\
Average & 1.00 & 0.44 & 0.00 & 0.00 \\
\hline
\end{tabular}




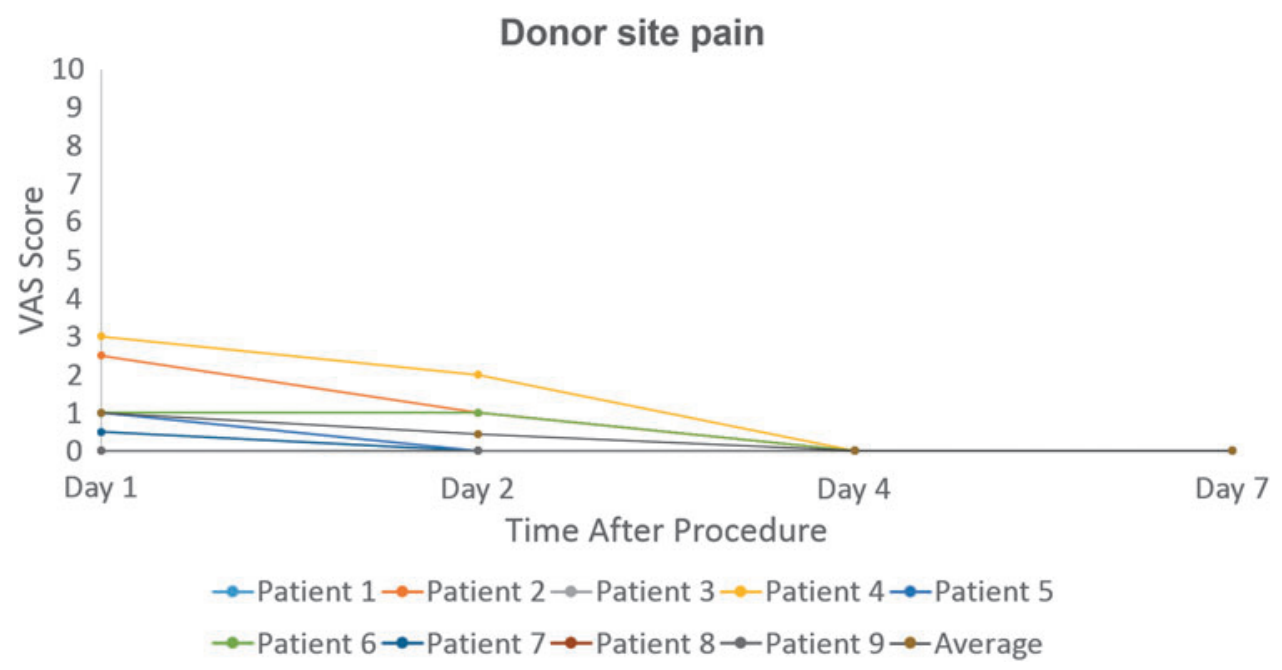

Figure 2. Patient VAS pain scores after treatment. VAS, visual analog scale.

after treatment was 1.0 ; on day 2 , the average VAS score was 0.4 (Fig. 2). None of the subjects recorded any pain on day 4 or later. The highest recorded pain of any patient was 3.0, documented $24 \mathrm{~h}$ after therapy. Two patients recorded the use of pain medication, but the medications were taken for conditions not associated with the donorsite pain.

\section{DISCUSSION}

When harvesting a graft, minimizing pain and ensuring rapid healing of the donor site wound are critical. Donor site pain is one of the most distressing symptoms reported by skin graft patients in the early postoperative period ${ }^{15}$ and is reported to impair patients' everyday activities. ${ }^{16}$ Several publications discuss the management of donor site wound care but fail to appropriately document the pain related to the harvesting of STSGs and FTSGs. It has been suggested that patients have difficulty reporting their donor site-related pain likely due to their perception of pain from the primary wound. ${ }^{17}$

Although autologous skin grafts are clinically proven to enhance wound healing, ${ }^{11}$ all skin grafting techniques are implicated in some degree of donor site trauma. ${ }^{18}$ Significant drawbacks of current harvesting techniques include pain at the donor site, limited donor skin availability, ${ }^{19}$ scarring, and an inability to restore complete skin function and architecture. ${ }^{12}$ There have been several attempts to reduce the trauma from donor site harvesting. New superficial harvesting techniques reduce donor area trauma; however, they may sacrifice therapeutic potential. It has been difficult to develop a harvesting technique that combines the advantages of FTSGs and little donor site morbidity.

The harvesting of FTSGs and STSGs can be a laborious and complex procedure usually obtained in a surgical setting. In our work, a low-complexity procedure for autologous FFTSG was used for restoration of a wide variety of wound etiologies. Our subjects reported minimal pain from graft harvesting. The average VAS 1 day after the procedure was 1.0. No pain was recorded on the fourth day and beyond.

FFTSG is a relatively simple procedure, performed in the outpatient setting without a surgical room or specialized dressings. Only local anesthesia and a single practitioner are necessary for this technique. These characteristics reduce the cost and regulatory complexity of the procedure. ${ }^{12}$

Tam et al. demonstrated that microcolumns comparably sized to those used in our study restore dermal and epidermal structure and function. These findings included a fully stratified epidermis, which contains melanocytes and Langerhans cells, and well-formed rete ridges. Furthermore, as seen with other FTSGs, dermal components such as fibroblasts, neurons, elastin fibers, hair follicles, and sweat glands were preserved at the recipient site. ${ }^{12}$ Transplanted adnexal structures, including the hair follicle bulge region and eccrine sweat glands, contain stem cells that have the potential to enhance the healing properties of the graft. ${ }^{20}$ The potential utility of deep dermal tissue and adnexal structures needs to be further explored.

Although this success in the restoration of skin function is encouraging, the focus of our study is the reduction of morbidity to the donor site. The concept behind this technology stems from frac- 
tional photothermolysis. This therapy delivers discreet micrometric columns of thermal injury that heal by remodeling and not scarring. ${ }^{13,21}$

In this report, we demonstrated that the harvester allows ease of procuring FFTSGs with minimal pain and morbidity at the donor site. Future research will need to evaluate other aspects of the procedure as well as long-term outcomes at the donor and recipient areas.

\section{KEY FINDINGS}

- FFTSG was associated with minimal donor-site pain.

- Nine subjects underwent the procedure and pain was evaluated using a validated numeric visual analog pain scale at days 1, 2, 4, and 7 after the procedure.

- Of the nine subjects, one-third reported no pain and none of the subjects reported pain after postoperation day 2 .

- The average pain recorded at days $1,2,4$, and 7 was $1.00,0.44,0.00$, and 0.00 , respectively.

\section{INNOVATION}

The utopian skin graft would harvest the most optimal and functional tissue with the least comorbidities in the donor site. To date, no skin grafting technique accomplishes these two tasks flawlessly. FFTSG is a new technology that could potentially yield a high-quality harvest with minor donor site complications. In our study, we tested pain at donor site after FFTSG. Of the nine patients who underwent this procedure, only three reported pain and no pain was documented after day 2 .

\section{ACKNOWLEDGMENTS AND FUNDING SOURCES}

Medline Industries provided the FFTSG device for the study. This article received no specific grant from any funding agency in the public, commercial, or not-for-profit sectors. Medline provided the harvester without charge.

\section{ABOUT THE AUTHORS}

Robert S. Kirsner, MD, PhD, is a Tenured Professor, Chairman, and holds the endowed Harvey Blank Chair in the Department of Dermatology and Cutaneous Surgery at the University of Miami Miller School of Medicine. He serves as Director of the University of Miami Hospital Wound Center and directs a wound healing clinical and transla- tional research team. Dr. Kirsner received his medical degree from the University of Miami. He completed a fellowship in wound healing, his residency in dermatology, and a doctorate in epidemiology from the same institution. Jose A. Jaller, MD, is currently serving as a postdoctoral research fellow in wound healing in the Department of Dermatology at the University of Miami. As part of his clinical research, Dr. Jaller serves as subinvestigator in numerous clinical trials, particularly studying healing of acute and chronic wounds. Hadar Lev-Tov, MD, is an assistant professor of dermatology at the University of Miami, where he leads multiple research projects. Drs. Ingrid Herskovitz, MD, Luis J. Borda, MD, and Penelope A. Hirt, MD, are postdoctoral research fellows in the Department of Dermatology at the University of Miami. Evan Darwin, BA, and Joshua Mervis, BA, are research fellows in the Department of Dermatology at the University of Miami.

\section{AUTHOR DISCLOSURE AND GHOSTWRITING}

Drs. Kirsner, Jaller, and Borda serve as clinical consultants for Medline Industries, Inc. The content of this article was expressly written by the authors listed. No ghostwriters were used to write this article.

\section{REFERENCES}

1. Lazarus GS, Cooper DM, Knighton DR, et al. Definitions and guidelines for assessment of wounds and evaluation of healing. Arch Dermatol 1994; 130:489-493.

2. Kelechi TJ, Johnson JJ, Yates S. Chronic venous disease and venous leg ulcers: an evidence-based update. J Vasc Nurs 2015;33:36-46.

3. Singer AJ, Tassiopoulos A, Kirsner RS. Evaluation and management of lower-extremity ulcers. N Engl J Med 2017;377:1559-1567.
4. Neville RF, Kayssi A, Buescher T, Stempel MS. The diabetic foot. Curr Probl Surg 2016;53:408437.

5. Lim HW, Collins SAB, Resneck JS, Jr., et al. The burden of skin disease in the United States. J Am Acad Dermatol. 2017;76:958-972.e2.

6. Kirsner RS, Eaglstein WH, Kerdel FA. Splitthickness skin grafting for lower extremity ulcerations. Dermatol Surg. 1997;23:85-91; quiz 2-3.
7. Kirsner RS, Falanga V, Eaglstein WH. The biology of skin grafts. Skin grafts as pharmacologic agents. Arch Dermatol 1993;129: 481-483

8. Herskovitz I, Hughes OB, Macquhae F, Rakosi A, Kirsner R. Epidermal skin grafting. Int Wound J 2016;13 Suppl 3:52-56.

9. Maderal AD, Kirsner RS. Clinical and economic benefits of autologous epidermal grafting. Cureus 2016;8:e875. 
10. Singh M, Nuutila K, Kruse C, Caterson EJ, Granter SR, Eriksson E. Fate of the dermal component of micrografts in full-thickness wounds. Eplasty 2014;14:e38.

11. Buchanan PJ, Kung TA, Cederna PS. Evidencebased medicine: wound closure. Plast Reconstr Surg 2014;134:1391-1404.

12. Tam J, Wang Y, Vuong LN, Fisher JM, Farinelli WA, Anderson RR. Reconstitution of full-thickness skin by microcolumn grafting. J Tissue Eng Regen Med 2017;11:2796-2805.

13. Tam J, Wang Y, Farinelli WA, et al. Fractional skin harvesting: autologous skin grafting without donor-site morbidity. Plast Reconstr Surg Global Open 2013;1:e47.

14. Hawker GA, Mian S, Kendzerska T, French M. Measures of adult pain: Visual Analog Scale for Pain (VAS Pain), Numeric Rating Scale for Pain (NRS Pain), McGill Pain Questionnaire (MPO), Short-Form McGill Pain Questionnaire (SF-MPO), Chronic Pain Grade Scale (CPGS), Short Form-36 Bodily Pain Scale (SF-36 BPS), and Measure of
Intermittent and Constant Osteoarthritis Pain (ICOAP). Arthritis Care Res (Hoboken) 2011;63 Suppl 11:S240-S252.

15. Sinha S, Schreiner AJ, Biernaskie J, Nickerson D, Gabriel VA. Treating pain on skin graft donor sites: review and clinical recommendations. J Trauma Acute Care Surg 2017;83:954-964.

16. Humrich M, Goepel L, Gutknecht M, et al. Healthrelated quality of life and patient burden in patients with split-thickness skin graft donor site wounds. Int Wound J 2018;15:266-273.

17. McBride CA, Kimble RM, Stockton K. Three donor site dressings in pediatric split-thickness skin grafts: study protocol for a randomised controlled trial. Trials 2015;16:43.

18. Svensjo T, Pomahac B, Yao F, Slama J, Wasif N, Eriksson E. Autologous skin transplantation: comparison of minced skin to other techniques. J Surg Res 2002;103:19-29.

19. Rettinger CL, Fletcher JL, Carlsson AH, Chan RK. Accelerated epithelialization and improved wound healing metrics in porcine full-thickness wounds transplanted with full-thickness skin micrografts. Wound Repair Regen 2017;25:816-827.

20. Jimenez F, Garde C, Poblet E, et al. A pilot clinical study of hair grafting in chronic leg ulcers. Wound Repair Regen 2012;20:806-814.

21. Manstein D, Herron GS, Sink RK, Tanner H, Anderson RR. Fractional photothermolysis: a new concept for cutaneous remodeling using microscopic patterns of thermal injury. Lasers Surg Med 2004:34:426-438.

\section{Abbreviations and Acronyms}

$\mathrm{DFU}=$ diabetic foot ulcer

$E S G=$ epidermal skin graft

FFTSG $=$ fractional autologous full-thickness skin grafting

FTSG $=$ full-thickness skin graft

STSG $=$ split-thickness skin graft

VAS $=$ visual analog scale

$\mathrm{VLU}=$ venous leg ulcer 